\title{
CFD Modelling of Pump as Turbine with Various Number of Blade for Microhydro System
}

\author{
Mohd Azlan Ismail ${ }^{1}$, Al Khalid Othman ${ }^{2}$, Hushairi Zen ${ }^{3}$, Mohd Suffian Misran ${ }^{4}$ \\ 1,2,3 Faculty of Engineering, University Malaysia Sarawak, Kota Samarahan, Malaysia. \\ ${ }^{4}$ Faculty of Engineering, University Malaysia Sabah, Kota Kinabalu, Malaysia. \\ ${ }^{1}$ Email:lanzmr@gmail.com, ${ }^{2}$ Email: okhalid@feng.unimas.my, \\ ${ }^{3}$ Email: zhushair@feng.unimas.my, Email: suffian@ums.edu.my
}

\begin{abstract}
Pump as Turbine is an electromechanically component that is largely used in microhydro system. The main advantages of pump as turbine compared to commercially available turbines are lower cost, easier to maintain and readily available. These key features make them appealing to conditions in many developing countries. However, pump as turbine has poor hydraulic performance and low efficiency thus modifications were applied to improve the performance. The aim of this paper is to study the effect of impeller blade number to the pump as turbine performance. The investigation was carried out by using commercial Computational Fluid Dynamic (CFD) software, ANSYS CFX. A centrifugal pump with a specific speed of 70 with an impeller diameter of $214.0 \mathrm{~mm}$ was used to generate the CAD model. The original number of the blade was at 6 and varied to 5, 7 and 8 while other geometric dimensions were kept unchanged. The simulation results reveal that the highest efficiency was attained at 7 blade number with the efficiency recorded at $76.24 \%$. The corresponding pressure was at 20.83 meters. It was found that with increase of blade number, the efficiency and corresponding pressure of the pump as turbine also increases. However, the additional blade number reduce the cross-area flow path, consequently increase the blockage effect thus decrease the net power generated by the rotating impeller.
\end{abstract}

Keywords: microhydro, pump as turbine, blade number, impeller, renewable energy

\section{Introduction}

Rural electrification has always been a central concern in many developing countries. It is widely recognized that electricity improves the quality of life by giving access to the basic services and infrastructure. The traditional approach to supply electricity to these areas is by extending the existing national high voltage transmission grid across hilly terrain and long distances, but this approach is prohibitively expensive. Off-grid, decentralized power generation systems are the alternatives to electrify small isolated communities [1]. There is a large number of energy generation systems such as standalone diesel generators, PV panels, wind turbine, biomass and hydropower systems. In some cases, two or more systems were combined to optimize the use of accessible resources.

Microhydro is the one of the best electric generation system and most cost effective among renewable energy systems. The microhydro system has the highest capacity factor compared to other types of energy generation systems thus it has the shortest return on investment and lowest life cycle

Manuscript History:

Received 20 August, 2015, Revised 7 March, 2016, Accepted 22 March, 2016, Published 31 March, 2016

e-ISSN 2289-7771

Copyright (C) 2016 JASPE 
cost [2]. However, traditional microhydro turbine such as Pelton, Turgo, Cross-flow, Francis and Kaplan turbine are expensive and difficult to manufacture due to the complex geometry profile. The use of end suction centrifugal pump in reverse flow named pump as turbine are frequently used to substitute commercially available turbine in many developing countries. The price of pump as turbine is lesser than traditional turbines thus reduce the initial capital cost due to mass-produced. On the other hand, pump as turbine has no flow adjust mechanism therefore less power can be harness at offdesign operation.

The number of blade is one of the important design features of the impeller. There can be a limited number of blade at finite blade thickness. The optimum number of blade depends on the operation scheme and requirements. The hydraulic loading on the impeller is the key governing factor that determines the optimum number of blade. There is a cut-off point, where the crowding-out effect increased up to a point, the total hydraulic loss overrides the net power produce by the impeller.

Sujoy, Pandey et al. (2012) presented a numerical analysis on the effect of blade number on centrifugal pumps at various rotational speeds using CFD numerical simulation [3]. The number of blade was varied from 4 to 12 . It was found that the pressure generated by the pump increased consistently with added number of blade. This result was supported by Liu Houlin et al. (2010) by presenting simulation and experimental analysis on a pump with a specific speed of 92.7 [4]. Furthermore, the simulation results highlighted the hydraulic field of jet and wake reduced with the increase number of blade, subsequently improve the hydraulic performance.

Splitter blades double the number of blade with additional smaller blade positioned circumferentially between the main blades minimizing the crowding effect at the impeller eye. Sheng et al. (2012) claimed that impeller with splitter blades increase pump s turbine efficiency [5]. The splitter blade increases the efficiency up to $3.42 \%$ and reduce the corresponding pressure from 40.69 to 36.80 meters, respectively. Sheng Yang et. al. (2012) investigated the influence of blade number to the pressure pulsation that normally linked to vibration and noise [6]. A centrifugal pump with a specific speed of 17 was selected as the study pump and the number of blade was adjusted from 6 to 9 . The highest efficiency was found at a blade number of 8 . The impeller with a number of blade at 9 was found to be the efficiency increase cut-off point, where the crowding-out loss starts to override the net energy produced by the impeller.

In order to further explore and extent understanding to the effect of blade number, this paper will present simulation analysis on a pump with specific speed of 70 . The sensitive parameters obtained from the simulation results are presented in characteristic curves.

\section{Research Methodology}

\subsection{Pump Specifications}

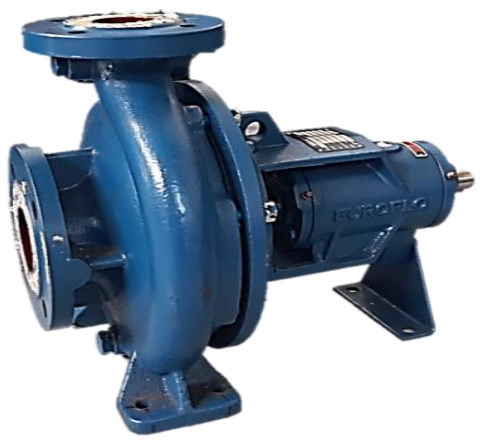

Figure 1: EuroFlo Pump with back pull feature 


\begin{tabular}{ccccc}
\hline \multicolumn{5}{c}{ Table 1: Pump Specifications } \\
\hline $\begin{array}{c}\text { Impeller diameter, D } \\
(\mathrm{mm})\end{array}$ & $\begin{array}{c}\text { Impeller height }, \mathrm{h} \\
(\mathrm{mm})\end{array}$ & $\begin{array}{c}\text { Wrap angle } \\
\text { (degrees) }\end{array}$ & $\begin{array}{c}\text { Eye Radius, } \mathrm{r} \\
(\mathrm{mm})\end{array}$ & $\begin{array}{c}\text { Number of } \\
\text { blades }\end{array}$ \\
\hline 214.0 & 48.0 & 160.0 & 84.2 & 6 \\
\hline
\end{tabular}

An end suction centrifugal pump with a specific speed of 70 was selected as the study pump, illustrated in Figure 1. The pump performance and dimension is according with BS EN733:1995; End suction centrifugal pump, rating at 10 bars with bearing bracket. The inlet and outlet diameter of the pump was recorded at $50.0 \mathrm{~mm}$ and $65.0 \mathrm{~mm}$, respectively. The best efficiency point of the pump was at $65.0 \%$ with corresponding pressure and flow rate of $14.0 \mathrm{~m}$ and $8.0 \mathrm{l} / \mathrm{s}$, respectively. Table 1 lists the main dimension of the pump under study.

\subsection{CAD Model and Mesh}

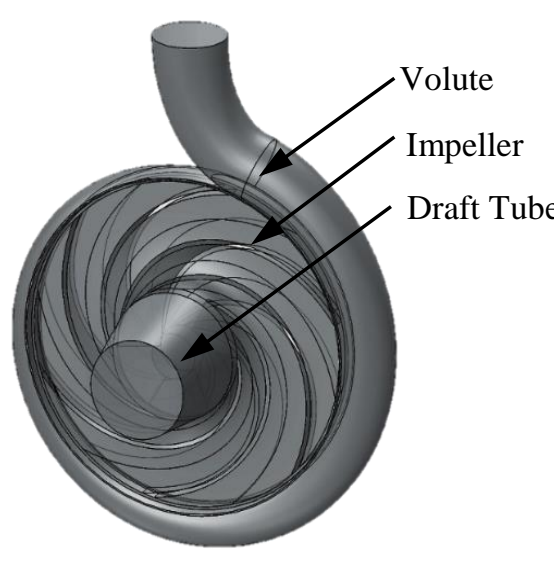

(a)

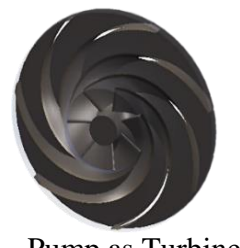

Pump as Turbine 7 Blades

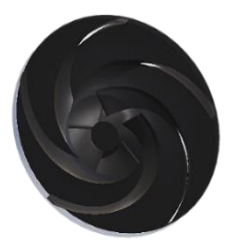

Pump as Turbine 5 Blades

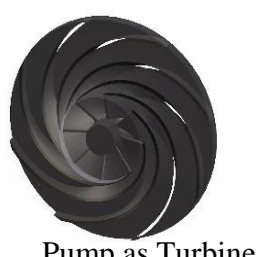

Pump as Turbine 8 Blades

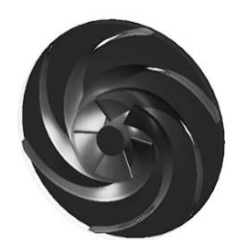

Pump as Turbine 6 Blades

(b)

Figure 2 (a) Pump as Turbine Computational Flow Domains

(b) Impeller with their respective number of blade

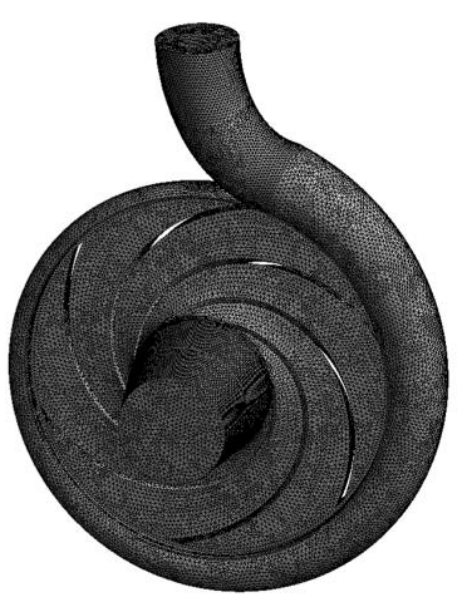

(a)

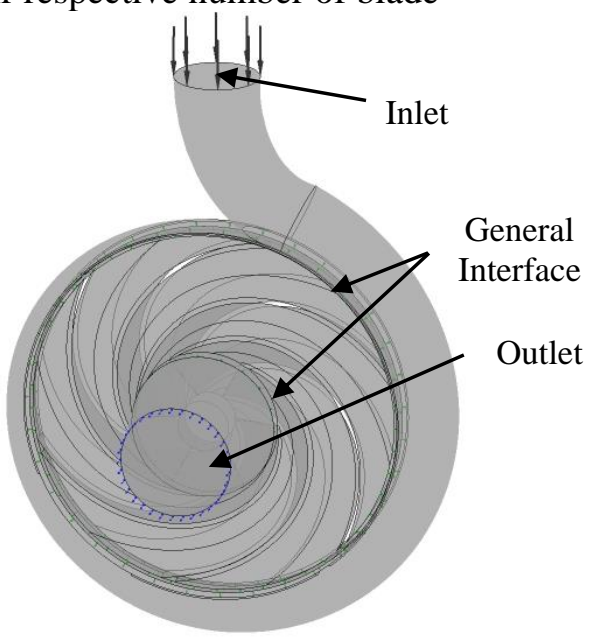

(b)

Figure 3 (a) Mesh topology at the domain surfaces

(b) Boundary conditions and interfaces 
The computational flow domains were divided into three distinct parts called volute, impeller and draft tube, as shown in Figure 2 (a). BladeGen in ANSYS Workbench was used as the CAD design tool to generate the impeller flow domain. The number of blade was varied to 5, 6, 7 and 8 , illustrated in Figure 2 (b).

The unstructured tetrahedral mesh was used to generate elements for the nodes inside the computational domains. The advantage of tetrahedral mesh is its flexibility in generating a satisfactory mesh shape and size for complex surfaces, particularly at the impeller. Mesh independence analysis was performed by refining the size of the mesh while observing the one sensitive parameter to evaluate mesh sensitivity. This procedure can help to determine the optimum balance between mesh resolutions and calculation time. The final mesh numbers for volute, impeller and draft tube were found at 450381, 476601 and 167226, respectively. The mesh topology for the flow domains is shown in Figure 3 (a).

\subsection{Boundary conditions and solver}

The governing Navier-Stokes equations based on the conservation laws for physical properties of fluids, which include mass, energy, and momentum. Standard k-epsilon turbulence model was selected that combine robustness with reasonable accuracy in turbomachinery analysis. The mathematical equations that were applied to the mass, momentum and energy can be expressed by the following equations:

Continuity Equation

$$
\frac{\partial \rho}{\partial t}+\nabla \bullet(\rho U)=0
$$

Momentum Equation

$$
\frac{\partial \rho U}{\partial t}+\nabla \bullet(\rho U \otimes U)=-\nabla p+\nabla \bullet \tau+S_{M}
$$

Momentum Equation with Rotational Forces

$$
\frac{\partial \rho U}{\partial t}+\nabla \bullet\left(\rho U \otimes U_{a b s}\right)=-\nabla \bullet\left(-p \delta+\mu\left(\nabla U+(\nabla U)^{T}\right)\right)-\rho \omega \times U-\rho \omega \times(\omega \times r)
$$

Energy Equation

$$
\frac{\partial\left(\rho h_{t o t}\right)}{\partial t}-\frac{\partial p}{\partial t}+\nabla \bullet\left(\rho U h_{t o t}\right)=\nabla \bullet(\lambda \nabla T)+\nabla \bullet(U \bullet \tau)+U \bullet S_{M}+S_{E}
$$

where, fluid density, $\rho$ time, $t$, vector of velocity, $U$, pressure, $p$, identity matrix, $\square$, viscosity, $\mu$ temperature, $T$, angular velocity, $\omega$, location vector, $r$, specific enthalpy, $h$, thermal conductivity, $\lambda$, shear stress, $\tau$, mass source, $S_{M}$, energy source, $S_{E}$

The working fluid was set as single-phase water, with a density of $1000 \mathrm{~kg} / \mathrm{m}^{3}$ at a temperature of $25{ }^{\circ} \mathrm{C}$. The boundary conditions were assigned to their respective physical boundaries; mass flow rate at the inlet, opening with atmospheric pressure reference at the outlet, while all other surfaces were set as wall surfaces at a surface roughness of 100 microns. The interfaces connecting the domains were set as general connections for both volute-impeller and impeller-draft tube as shown in 
Figure 3(b). The mass flow rate was varied from $10.0 \mathrm{l} / \mathrm{s}$ to $18.0 \mathrm{l} / \mathrm{s}$. The rotational speed of the impeller was set to 1550 RPM.

\section{Result and Discussion}

Table 2: Pump as Turbine best efficient point at $14.0 \mathrm{l} / \mathrm{s}$

\begin{tabular}{cccc}
\hline Blade Number & $\mathrm{H}_{\text {BEP }}(\mathrm{m})$ & $\mathrm{P}_{\text {BEP }}(\mathrm{kW})$ & $\eta(\%)$ \\
\hline 5 & 20.63 & 2.15 & 75.85 \\
6 & 20.46 & 2.13 & 75.77 \\
7 & 20.83 & 2.18 & 76.24 \\
8 & 21.10 & 2.19 & 75.57 \\
\hline
\end{tabular}
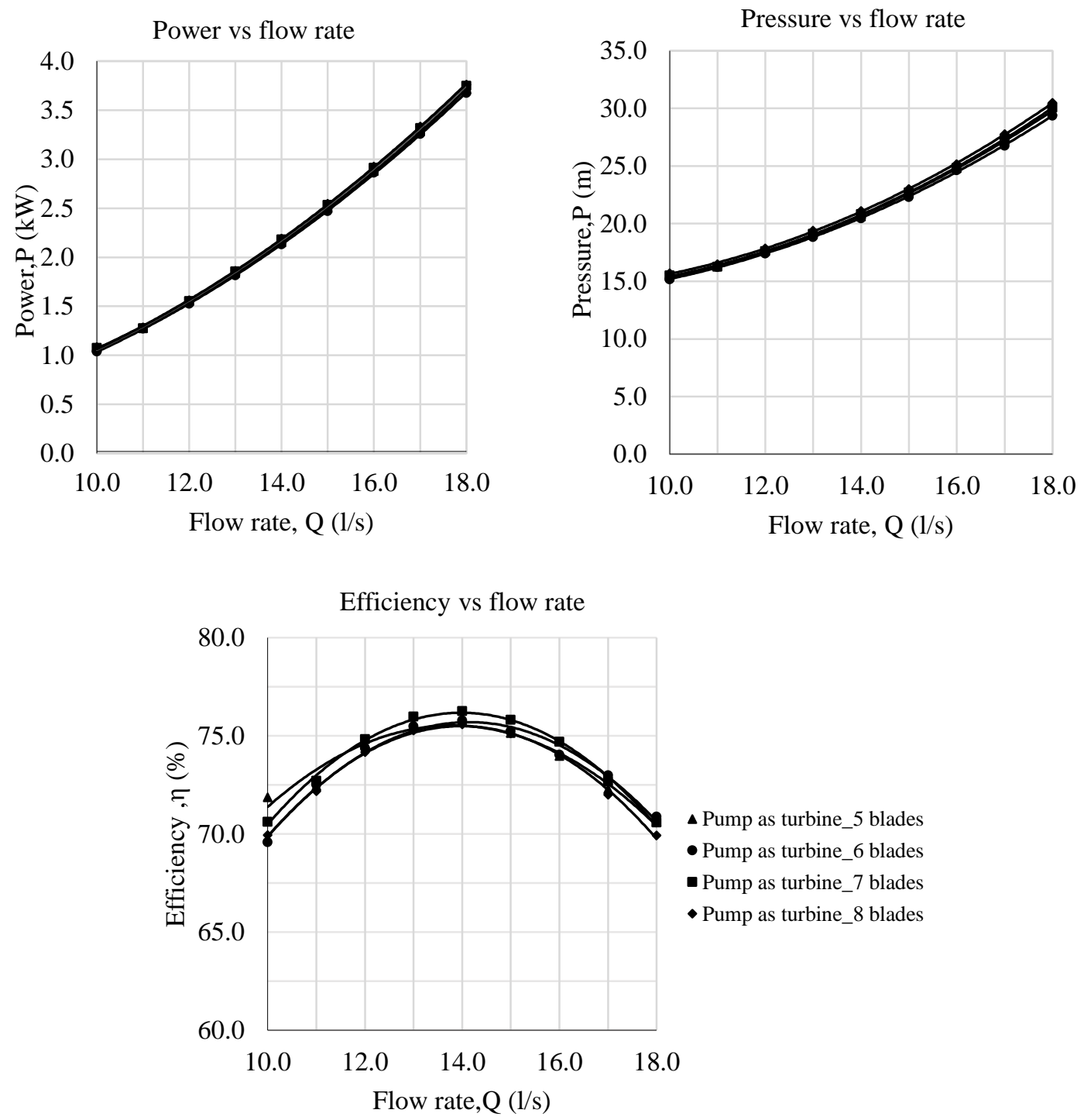

Figure 4: Pump as Turbine Performance Curve

The CFD results are presented in performance curve diagram illustrated in Figure 4. The diagram shows the pressure, power and efficiency at possible operating flow range. The summary of best efficient points is shown in Table 2 . 
The performance curves illustrate a typical fixed blade radial-type turbine operation. It can be observed that the efficiency curve has a negative quadratic profile; steep drop at part-load and overload operation. The pressure increase with the increase of flow rate.

All the impellers has similar performance curve profile. The best efficient points were recorded at $14.0 \mathrm{l} / \mathrm{s}$. It should be noted that higher number of blade increase the corresponding operation pressure at best efficient point despite the flow retained its value at $14.01 / \mathrm{s}$. Furthermore, the power generated by the impeller increase as the blade number increases.

The efficiencies at BEP are $75.85 \%, 75.77 \%, 76.24 \%$ and $75.57 \%$ for blade number of $5,6,7$ and 8 respectively. The highest efficiency was recorded at 7 blade number, with efficiency and pressure of $76.24 \%$ and 20.83 meter respectively. Impeller with 8 blade number is the cut-off point where the efficiency start to reduce to $75.57 \%$. This indicates that effective blockage effect has increased consequently reduce the net hydraulic power impart to the impeller blade.

\section{Conclusion}

In this paper, the effect of blade number on Pump as Turbine performance was analyzed by using ANSYS CFX software. One end centrifugal pump with a specific speed of 70 was selected as the study pump for the analysis. The number of blade was varied to 5, 6, 7 and 8 . It was found that all the impellers have a similar performance curve profile. The simulation result showed that the highest efficiency was generated by the impeller blade number of 7 .

\section{Reference}

[1] "ASEAN Guideline on Off-grid Rural Electrification Approaches," 2013.

[2] O. A. Jaramillo, M. A. Borja, and J. M. Huacuz, "Using Hydropower to Complement Wind Energy: A Hybrid System to Provide Firm Power," Renewable Energy, vol. 29, pp. 1887-1909, 2004.

[3] S. Chakraborty, K. M. Pandey, and B. Roya, "Numerical Analysis on Effects of Blade Number Variations on Performance of Centrifugal Pumps with Various Rotational Speeds," International Journal of Current Engineering and Technology, vol. 2, 2012.

[4] Liu Houlin, W. Yong, Yuan Shouqi, T. Minggao, and Wang Kai, "Effects of Blade Number on Characteristics of Centrifugal Pumps," Chinese Journal of Mechanical Engineering, vol. 23, 2010.

[5] Y. Sun-Sheng, K. Fan-Yu, F. Jian-Hui, and X. Ling, "Numerical Research on Effects of Splitter Blades to the Influence of Pump as Turbine," International Journal of Rotating Machinery, 2012.

[6] S. S. Yang, F. Y. Kong, X. Y. Qu, and W. M. Jiang, "Influence of Blade Number on the Performance and Pressure Pulsations in a Pump Used as a Turbine," Journal of Fluids Engineering, Transactions of the ASME, vol. 134, 2012. 


\section{Authors}

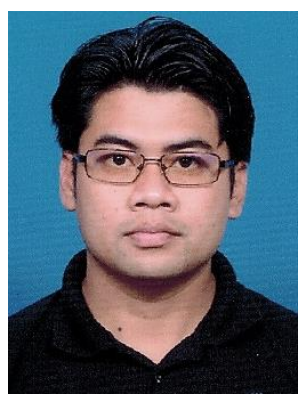

\section{Mr. Mohd Azlan Ismail}

Mohd Azlan Ismail is a PhD candidate in Mechanical Engineering at University Malaysia Sarawak, Malaysia. His research interest is in renewable energy focused on microhydro power for rural electrification. He graduated from University Malaysia Sabah and has a Master Degree in Mechanical Engineering Practice from University of Wollongong, Australia. Mohd Azlan Ismail is a graduate member of Institute of Engineers, Malaysia.

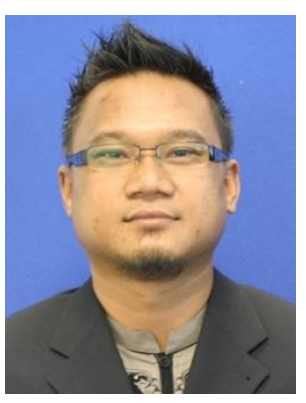

\section{Dr. Al Khalid Othman}

Dr. A.K. Othman was born on July 12, 1971, in Kuching Sarawak, Malaysia. He is graduated his first degree in BEng (Hons) in Electrical and Electronic Engineering in Nottingham Trent University, U.K in 1995 and Masters in Information Technology (Digital) at Nottingham University, U.K. in $1996 . \mathrm{He}$ gained $\mathrm{PhD}$ in Engineering, specialized in Underwater Acoustic Network at Newcastle University, U.K. in 2007. He became a Member (M) of Board of Engineers Malaysia since 16 November 1999. His research interests include underwater communication, wireless protocol, networking and renewable energy.

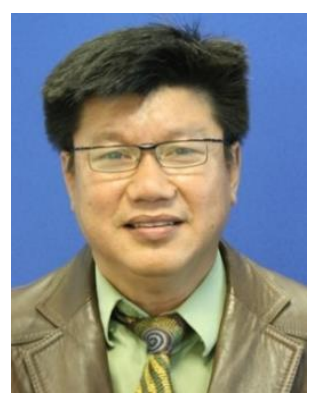

\section{Dr. Hushairi Zen}

Dr. Hushairi Zen obtained his Doctor of Philosophy $(\mathrm{PhD})$ in 2009 at Edith Cowan University, Perth in Wireless Local Area Network. His area of expertise include wireless communication, data and voice networking, remote monitoring and control, and renewable energy. He has published more than 30 papers on the subject of his interest and also written 4 books. He is currently supervising $4 \mathrm{PhD}$ and 5 Master students.

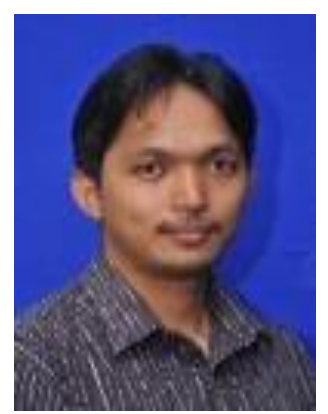

\section{Mohd Suffian Misran}

Mohd Suffian is a Lecturer in the Faculty of Engineering at Universiti Malaysia Sabah. He completed his bachelor's degree in Mechanical Engineering and his master degree from Universiti Teknologi Malaysia in 2001 and 2007 respectively. At the time, he also involves himself with research and development activity mainly on HVAC and compressors. He is the author of several engineering papers varying from design, statistical analysis, membrane, CFD, thermal and renewable energy. He also involves in a number of research grants related to renewable energy either as project leader or research associates. 